International Mathematical Forum, 2, 2007, no. 29, 1421 - 1429

\title{
Sensitivity Analysis of Efficient Units in Data Envelopment Analysis
}

\author{
G. R. Jahanshahloo \\ Dept. of Math., Science and Research Branch, \\ Islamic Azad University, Tehran 14515-775, Iran \\ F. Hosseinzadeh Lotfi $^{1}$ \\ Dept. of Math., Science and Research Branch, \\ Islamic Azad University, Tehran 14515-775, Iran \\ M. Esmaeili \\ Dept. of Math., Islamic Azad University, \\ Shahrekord, tel: +98-381-3330722, Iran

\section{Mohammad Izadikhah} \\ Dept. of Math., Islamic Azad University, Arak, \\ tel:+98-861-3663041-9, P.O. Box 38135/567, Iran
}

\begin{abstract}
In data envelopment analysis (DEA) efficient decision making units (DMUs) are of primary importance as they define the efficient frontier. By means of modified CCR model, in which the test DMU is excluded from the reference set, we are able to determine what perturbations of data can be tolerated before frontier DMUs become nonfrontier. In this paper we discuss simultaneous data perturbations in all DMUs, where the efficiency of the test DMU is deteriorating while the efficiencies of the other DMUs are improving. Necessary and sufficient conditions for preserving a DMU's efficiency classification presented by Zhu [8] are developed and improved when various data changes are applied to all DMUs. The region, for preserving a DMU's classification, that is, greater than the region presented in Zhu [8] is then calculated.
\end{abstract}

\footnotetext{
${ }^{1}$ Corresponding author:Farhad Hosseinzadeh Lotfi, E-mail: hosseinzadeh_lotfi@yahoo.com
} 
Mathematics Subject Classification: Operations Research, No. 90

Keywords: Data envelopment analysis(DEA); Efficiency; Sensitivity analysis; Super efficiency

\section{Introduction}

Data envelopment analysis(DEA), as developed by Charnes et al.[2](CCR), is a methodology based on a linear programming (LP) model for evaluating relative efficiencies of DMUs with common inputs and outputs. One important issue in DEA which has been studied by many DEA researchers is sensitivity analysis of a specific $D M U_{o}$, unit under evaluation. We are interested in sensitivity analysis of frontier DMUs based on Charnes et al.(1991) consist of DMUs in set $\mathbf{E}$ (extreme efficient), set $\mathbf{E}^{\prime}$ (efficient but not an extreme efficient) and set $\mathbf{F}$ (weakly efficient). One type of DEA sensitivity analysis is based on super-efficiency DEA approach in which a test DMU is not included in reference set(Andersen and Petersen, [1]; Seiford and Zhu [6]). Charnes et al.[4] and Charnes et al.[5] develop a super-efficiency DEA sensitivity analysis technique for the situation where simultaneous proportional change is assumed in all inputs and outputs for a test DMU.Seiford and Zhu[6] and Zhu[8] generalize the technique in charnes et al.[4 ] and Charnes et al.[5] to the worst-case scenario where the efficiency of the test DMU is deteriorating while the efficiencies of the other DMUs are improving. In his method, simultaneous data perturbations in input/output of $D M U_{j}(j \neq o)$ and $D M U_{o}$ is assumed and necessary and sufficient conditions for preserving a DMU's efficiency classification are developed. In this paper we develop and improve the necessary and sufficient conditions for preserving a DMU's efficiency presented in Zhu(2001) when data changes are applied to all DMUs .

The structure of the paper is as follows : in section 2, we introduce simultaneous data changes in all data and express the necessary and sufficient conditions for preserving efficiency of a frontier $D M U_{o}$ presented in Zhu[8], in sections 3 and 4 , we develop and improve necessary and sufficient conditions for preserving efficiency of a frontier $D M U_{o}$, conclusions are given in section 5.

\section{Simultaneous changes in all the data}

Suppose we have a set of $\mathrm{n}$ DMUs . Each $D M U_{j}(j=1, \ldots, n)$ produces $\mathrm{s}$ different outputs $y_{r j}(r=1, \ldots, s)$ utilizing $m$ different inputs $x_{i j}(i=1, \ldots, m)$. We assume that all inputs and outputs are positive. Let $\mathbf{I}$ and $\mathbf{O}$ denote respectively the input and output subsets in which we are interested. That is 
, we consider the data changes in set $\mathbf{I}$ and set $\mathbf{O}$. Then consider percentage data perturbations presented in Zhu(2001) as follows:

for $D M U_{o}$ :

$$
\begin{array}{cl}
\hat{x}_{i o}=\delta_{i} x_{i o} \quad \delta_{i} \geq 1, & i \in \mathbf{I} \\
\hat{x}_{i o}=x_{i o}, & i \notin \mathbf{I} \\
\hat{y}_{r o}=\tau_{r} y_{r o}, \quad 0<\tau_{r} \leq 1, & r \in \mathbf{O} \\
\hat{y}_{r o}=y_{r o}, & r \notin \mathbf{O}
\end{array}
$$

for $D M U_{j}(j \neq o)$ :

$$
\begin{aligned}
& \hat{x}_{i j}=\frac{x_{i j}}{\tilde{\delta}_{i}}, \quad \widetilde{\delta}_{i} \geq 1, \quad i \in \mathbf{I} \\
& \hat{x}_{i j}=x_{i j}, \quad i \notin \mathbf{I} \\
& \hat{y}_{r j}=\frac{y_{r j}}{\tau_{r}}, \quad 0<\widetilde{\tau}_{r} \leq 1, \quad r \in \mathbf{O} \\
& \hat{y}_{r j}=y_{r j}, \quad r \notin \mathbf{O}
\end{aligned}
$$

where ( )represents adjusted data.

We consider the following modified DEA measure presented in Zhu(2001) for simultaneous variations of inputs and outputs :

$$
\begin{aligned}
& \Gamma^{*}=\min \Gamma \\
& \text { s.t. } \quad \sum_{j=1, j \neq o}^{n} \lambda_{j} x_{i j} \leq(1+\Gamma) x_{i o}, \quad i \in \mathbf{I} \\
& \sum_{j=1, j \neq o}^{n} \lambda_{j} x_{i j} \leq x_{i o}, \quad i \notin \mathbf{I} \\
& \sum_{\substack{j=1, j \neq o \\
n}}^{n} \lambda_{j} y_{r j} \geq(1-\Gamma) y_{r o}, \quad r \in \mathbf{O} \\
& \sum_{j=1, j \neq o}^{n} \lambda_{j} y_{r j} \geq y_{r o}, \quad r \notin \mathbf{O} \\
& \lambda_{j} \geq 0 \quad, j=1, \ldots, n,(j \neq o)
\end{aligned}
$$

Note that if $D M U_{o}$ is a frontier point, then $\Gamma \geq 0$. Model(1) for evaluating 
$D \widehat{M U} U_{o}$ turns in to :

$$
\begin{aligned}
& \widehat{\Gamma}^{*}=\min \hat{\Gamma} \\
& \text { s.t. } \sum_{j=1, j \neq o}^{n} \lambda_{j} \frac{x_{i j}}{\widetilde{\delta}_{i}} \leq(1+\widehat{\Gamma}) \delta_{i} x_{i o}, \quad i \in \mathbf{I} \\
& \sum_{j=1, j \neq o}^{n} \lambda_{j} x_{i j} \leq x_{i o}, \quad i \notin \mathbf{I} \\
& \sum_{j=1, j \neq o}^{n} \lambda_{j} \frac{y_{r j}}{\widetilde{\tau}_{r}} \geq(1-\widehat{\Gamma}) \tau_{r} y_{r o}, \quad r \in \mathbf{O} \\
& \sum_{j=1, j \neq o}^{n} \lambda_{j} y_{r j} \geq y_{r o}, \quad r \notin \mathbf{O} \\
& \lambda_{j} \geq 0 \quad, j=1, \ldots, n,(j \neq o)
\end{aligned}
$$

Zhu presented the sufficient conditions for preserving efficiency of a frontier $D M U_{o}$ as follows (Theorem 5 in $\mathrm{Zhu}(2001)$ ):

Theorem 1 : Suppose $D M U_{o}$ is a frontier point. If $1 \leq \delta_{i} \widetilde{\delta}_{i} \leq \sqrt{1+\Gamma^{*}}, i \in \mathbf{I}$ and $\sqrt{1-\Gamma^{*}} \leq \tau_{r} \widetilde{\tau}_{r} \leq 1, r \in \mathbf{O}$ then $D M U_{o}$ remains as a frontier point, where $\Gamma^{*}$ is the optimal value to (1).

Zhu also presented the following necessary conditions for preserving extreme efficiency: For an extreme-efficient $D M U_{o}$, if $\delta_{i} \widetilde{\delta}_{i}>\sqrt{1+\Gamma^{*}}, i \in \mathbf{I}$ and $\tau_{r} \widetilde{\tau}_{r}<\sqrt{1-\Gamma^{*}}, r \in \mathbf{O}$ then $D M U_{o}$ will not remain as a extreme point.

In the next section, first, with a counter example we show that the above mentioned are not correct. And we modify the necessary and sufficient conditions presented in Zhu (2001) .

\section{Counter example violating correctness of the necessary conditions presented}

Example : Consider the four DMUs A,B,C and D with two inputs and one single output in table 1 . We know that A belong to set $\mathbf{E}$.

Table 1: Sample DMUs (Before perturbations)

\begin{tabular}{lllll}
\hline $\mathrm{DMU}$ & $\mathrm{y}$ & $x_{1}$ & $x_{2}$ & $\theta_{C C R}^{*}$ \\
\hline $\mathrm{A}$ & 1 & 2 & 2 & 1 \\
$\mathrm{~B}$ & 1 & $\frac{6}{5}$ & 6 & 1 \\
$\mathrm{C}$ & 1 & 6 & $\frac{6}{5}$ & 1 \\
$\mathrm{D}$ & 1 & 3 & 3 & $\frac{2}{3}$ \\
\hline
\end{tabular}


First we solve model(1) for $\mathrm{A}$, then we have $\Gamma_{A}^{*}=\frac{1}{2}$. (In this situation we assume $\mathbf{I}=\{1,2\}$ and $\mathbf{O}=)$. Then by choosing $\delta_{i}=\frac{5}{4}$ and $\widetilde{\delta}_{i}=\frac{12}{10}, i \in \mathbf{I}$ we have $\delta_{i} \widetilde{\delta}_{i}=\frac{3}{2}=1+\frac{1}{2}=1+\Gamma_{A}^{*}>\sqrt{1+\Gamma_{A}^{*}}=\sqrt{\frac{3}{2}}$, but $\widehat{A}$ remains extremeefficient.(table $(2))$.

Table 2 : After perturbations,

\begin{tabular}{lllll}
\hline DMU & $\mathrm{y}$ & $x_{1}$ & $x_{2}$ & $\theta_{C C R}^{*}$ \\
\hline$\widehat{A}$ & 1 & $\frac{5}{2}$ & $\frac{5}{2}$ & 1 \\
$\widehat{B}$ & 1 & 1 & 5 & 1 \\
$\widehat{C}$ & 1 & 5 & 1 & 1 \\
$\widehat{D}$ & 1 & $\frac{5}{2}$ & $\frac{5}{2}$ & 1 \\
\hline
\end{tabular}

\section{Modification of the sufficient and necessary conditions presented by Zhu}

We modify the sufficient conditions for preserving efficiency as the following theorem :

Theorem 2: Suppose $D M U_{o}$ is a frontier DMU . If $1 \leq \delta_{i} \widetilde{\delta}_{i} \leq 1+\Gamma^{*}, i \in \mathbf{I}$ and $1-\Gamma^{*} \leq \tau_{r} \widetilde{\tau}_{r} \leq 1, r \in \mathbf{O}$ then $D M U_{o}$ remains as a frontier point, where $\Gamma^{*}$ is the optimal value to $(1)$.

Proof: By contradiction we assume that $D \widehat{M} U_{o}$ is not a frontier point. Suppose that $\widehat{\Gamma}^{*}$ be the optimal value of model(2), Then by assumption we have $\widehat{\Gamma}^{*}<0$.

At optimality, the constraints of $\operatorname{model}(2)$ is as follows: 


$$
\begin{aligned}
& \sum_{j=1, j \neq o}^{n} \widehat{\lambda}_{j}^{*} x_{i j} \leq\left(1+\widehat{\Gamma}^{*}\right) \delta_{i} \widetilde{\delta}_{i} x_{i o}, \quad i \in \mathbf{I} \\
& \sum_{j=1, j \neq o}^{n} \hat{\lambda}_{j}^{*} x_{i j} \leq x_{i o}, \quad i \notin \mathbf{I} \\
& \sum_{j=1, j \neq o}^{n} \widehat{\lambda}_{j}^{*} y_{r j} \geq\left(1-\widehat{\Gamma}^{*}\right) \tau_{r} \widetilde{\tau}_{r} y_{r o}, \quad r \in \mathbf{O} \\
& \sum_{j=1, j \neq o}^{n} \hat{\lambda}_{j}^{*} y_{r j} \geq y_{r o}, \quad r \notin \mathbf{O}
\end{aligned}
$$

Then we have:

$$
\begin{array}{ll}
\sum_{j=1, j \neq o}^{n} \hat{\lambda}_{j}^{*} x_{i j} \leq\left(1+\left(\left(1+\widehat{\Gamma}^{*}\right) \delta_{i} \widetilde{\delta}_{i}-1\right)\right) x_{i o}, & i \in \mathbf{I} \\
\sum_{j=1, j \neq o}^{n} \hat{\lambda}_{j}^{*} x_{i j} \leq x_{i o}, & i \notin \mathbf{I} \\
\sum_{\substack{n=1, j \neq o \\
n}}^{n} \hat{\lambda}_{j}^{*} y_{r j} \geq\left(1-\left(1-\left(1-\widehat{\Gamma}^{*}\right) \tau_{r} \widetilde{\tau}_{r}\right)\right) y_{r o}, & r \in \mathbf{O} \\
\sum_{j=1, j \neq o}^{n} \hat{\lambda}_{j}^{*} y_{r j} \geq y_{r o}, & r \notin \mathbf{O}
\end{array}
$$

Let:

$\widehat{\gamma}=\max \left\{\max \left\{\left(1+\widehat{\Gamma}^{*}\right) \delta_{i} \widetilde{\delta}_{i}-1, i \in \mathbf{I}\right\}, \max \left\{1-\left(1-\widehat{\Gamma}^{*}\right) \tau_{r} \widetilde{\tau}_{r}, r \in \mathbf{O}\right\}\right\}$

Then,we have:

$\widehat{\gamma} \geq\left(\left(1+\widehat{\Gamma}^{*}\right) \delta_{i} \widetilde{\delta}_{i}-1\right), i \in \mathbf{I}$ and $\widehat{\gamma} \geq\left(1-\left(1-\widehat{\Gamma}^{*}\right) \tau_{r} \widetilde{\tau}_{r}\right), r \in \mathbf{O}$

Thus we have :

$$
\begin{array}{ll}
\sum_{j=1, j \neq o}^{n} \widehat{\lambda}_{j}^{*} x_{i j} \leq(1+\widehat{\gamma}) \delta_{i} \widetilde{\delta}_{i} x_{i o}, & i \in \mathbf{I} \\
\sum_{j=1, j \neq o}^{n=1, n} \widehat{\lambda}_{j}^{*} x_{i j} \leq x_{i o}, & i \notin \mathbf{I} \\
\sum_{j=1, j \neq o}^{n} \widehat{\lambda}_{j}^{*} y_{r j} \geq(1-\widehat{\gamma}) \tau_{r} \widetilde{\tau}_{r} y_{r o}, & r \in \mathbf{O} \\
\sum_{j=1, j \neq o}^{n} \hat{\lambda}_{j}^{*} y_{r j} \geq y_{r o}, & r \notin \mathbf{O}
\end{array}
$$

Then $\left(\widehat{\gamma}, \hat{\lambda}_{j}^{*},(j \neq o)\right)$ is a feasible solution to $(1)$. Therefore $\widehat{\gamma} \geq \Gamma^{*}$. We have the two following cases :

Case I: If $\widehat{\gamma}=\max \left\{\left(1+\widehat{\Gamma}^{*}\right) \delta_{i} \widetilde{\delta}_{i}-1, i \in \mathbf{I}\right\}=\left(1+\widehat{\Gamma}^{*}\right) \delta_{k} \widetilde{\delta}_{k}-1$, then by assumption since $1 \leq \delta_{i} \widetilde{\delta}_{i} \leq 1+\Gamma^{*}, i \in \mathbf{I}$, then we have $\delta_{k} \widetilde{\delta}_{k} \leq 1+\Gamma^{*}$. Since $\widehat{\Gamma}^{*}<0$ then $0<1+\widehat{\Gamma}^{*}<1$. Thus we have $\left(1+\widehat{\Gamma}^{*}\right) \delta_{k} \widetilde{\delta}_{k} \leq\left(1+\widehat{\Gamma}^{*}\right)\left(1+\Gamma^{*}\right)<$ $\left(1+\Gamma^{*}\right)$. Its means that $\hat{\gamma}<\Gamma^{*}$. This leads to a contradiction with $\widehat{\gamma} \geq \Gamma^{*}$.

Case II: If $\widehat{\gamma}=\max \left\{\left(1-\left(1-\widehat{\Gamma}^{*}\right) \tau_{r} \widetilde{\tau}_{r}\right), r \in \mathbf{O}\right\}=\left(1-\left(1-\widehat{\Gamma}^{*}\right) \tau_{p} \widetilde{\tau}_{p}\right.$ then by assumption since $1-\Gamma^{*} \leq \tau_{r} \widetilde{\tau}_{r} \leq 1, r \in \mathbf{O}$, then we have $\tau_{p} \widetilde{\tau}_{p} \geq 1-\Gamma^{*}$. Since $\widehat{\Gamma}^{*}<0$ then $1-\widehat{\Gamma}^{*}>1$. Thus we have $\left(1-\widehat{\Gamma}^{*}\right)\left(\tau_{p} \widetilde{\tau}_{p}\right) \geq\left(1-\widehat{\Gamma}^{*}\right)\left(1-\Gamma^{*}\right)>$ 
$\left(1-\Gamma^{*}\right)$. Its means that $\hat{\gamma}<\Gamma^{*}$. This leads to a contradiction with $\widehat{\gamma} \geq \Gamma^{*}$ .Therefore $D \widehat{M} U_{o}$ remains as a frontier point.

Before modifying the necessary conditions for preserving extreme efficiency, we need the following lemma:

Lemma: In the optimal solution of model(2), an equality exists at least in one of the constraints (2.1)or(2.3).

Proof: Let $\left(\bar{\Gamma}, \bar{\lambda}_{j},(j \neq o)\right)$ be the optimal solution of model(2).By contradiction, we assume that there is no equality in any of the above constraints,

that is, we have : $\quad \sum_{\substack{j=1, j \neq o \\ n}}^{n} \bar{\lambda}_{j} x_{i j} \leq x_{i o}, \quad i \notin \mathbf{I}$

that is, we have : ${ }_{n}$ then we have :

$$
\begin{array}{ll}
\sum_{j=1, j \neq o}^{n} \bar{\lambda}_{j} x_{i j}<(1+\bar{\Gamma}) \delta_{i} \widetilde{\delta}_{i} x_{i o}, & i \in \mathbf{I} \\
\sum_{\substack{n=1, j \neq o \\
n}}^{n} \bar{\lambda}_{j} x_{i j} \leq x_{i o}, & i \notin \mathbf{I} \\
\sum_{\substack{j=1, j \neq o \\
n}} \bar{\lambda}_{j} y_{r j}>(1-\bar{\Gamma}) \tau_{r} \widetilde{\tau}_{r} y_{r o}, \quad r \in \mathbf{O}
\end{array}
$$$$
\sum_{j=1, j \neq o}^{n} \bar{\lambda}_{j} y_{r j} \geq y_{r o}, \quad \quad r \notin \mathbf{O}
$$

$$
\begin{aligned}
& 1+\bar{\Gamma}>\frac{\sum_{j=1, j \neq o}^{n} \bar{\lambda}_{j} x_{i j}}{\delta_{i} \widetilde{\delta}_{i} x_{i o}}, \quad i \in \mathbf{I} \quad \text { or equivalently : } \quad \bar{\Gamma}>\frac{\sum_{j=1, j \neq o}^{n} \bar{\lambda}_{j} x_{i j}}{\delta_{i} \widetilde{\delta}_{i} x_{i o}}-1, \quad i \in \mathbf{I} \\
& 1-\bar{\Gamma}<\frac{\sum_{j=1, j \neq o}^{n} \bar{\lambda}_{j} y_{r j}}{\tau_{r} \tau_{r} y_{r o}}, r \in \mathbf{O} \quad \bar{\Gamma}>1-\frac{\sum_{j=1, j \neq o}^{n} \bar{\lambda}_{j} y_{r j}}{\tau_{r} \tau_{r} y_{r o}}, r \in \mathbf{O}
\end{aligned}
$$

Obviously, $\bar{\Gamma}>\alpha$. Define $\widetilde{\Gamma}=\frac{\bar{\Gamma}+\alpha}{2}$, then we have : $\alpha<\widetilde{\Gamma}<\bar{\Gamma}$ and obviously $\left(\widetilde{\Gamma}, \bar{\lambda}_{j},(j \neq o)\right)$ is a feasible solution to model $(2)$, violating the optimality of $\bar{\Gamma}$.

Theorem 3: Suppose $D M U_{o}$ is a extreme efficient . If $\delta_{i} \widetilde{\delta}_{i}>1+\Gamma^{*}, i \in \mathbf{I}$ and $\tau_{r} \widetilde{\tau}_{r}<1-\Gamma^{*}, r \in \mathbf{O}$ then $D M U_{o}$ will not remain extreme efficient.

Proof:First, we assume $\left(\widehat{\Gamma}^{*}, \widehat{\lambda}_{j},(j \neq o)\right)$ to be the optimal solution to model $(2)$. By contradiction, we assume that $D M U_{o}$ remains as extreme efficient, that is, $\widehat{\Gamma}^{*}>0$. Then we have : $\begin{array}{ll}1+\widehat{\Gamma}^{*}>1 \\ 1-\widehat{\Gamma}^{*}<1 .\end{array}$

At optimality constraints in model (1) can be expressed as : 


$$
\begin{array}{ll}
\sum_{j=1, j \neq o}^{n} \lambda_{j}^{*} x_{i j} \leq\left(1+\Gamma^{*}\right) x_{i o}, & i \in \mathbf{I} \\
\sum_{j=1, j \neq o}^{n} \lambda_{j}^{*} x_{i j} \leq x_{i o}, & i \notin \mathbf{I} \\
\sum_{\substack{j=1, j \neq o \\
n}}^{n} \lambda_{j}^{*} y_{r j} \geq\left(1-\Gamma^{*}\right) y_{r o}, & r \in \mathbf{O} \\
\sum_{j=1, j \neq o}^{n} \lambda_{j}^{*} y_{r j} \geq y_{r o}, & r \notin \mathbf{O}
\end{array}
$$

By assumption, the above constraints turn in to :

$$
\begin{array}{ll}
\sum_{j=1, j \neq o}^{n} \lambda_{j}^{*} x_{i j} \leq\left(1+\Gamma^{*}\right) x_{i o}<\delta_{i} \widetilde{\delta}_{i} x_{i o}<\left(1+\widehat{\Gamma}^{*}\right) \delta_{i} \widetilde{\delta}_{i} x_{i o}, & i \in \mathbf{I} \\
\sum_{j=1, j \neq o}^{n} \lambda_{j}^{*} x_{i j} \leq x_{i o}, & i \notin \mathbf{I} \\
\sum_{\substack{j=1, j \neq o \\
n}}^{n} \lambda_{j}^{*} y_{r j} \geq\left(1-\Gamma^{*}\right) y_{r o}>\tau_{r} \widetilde{\tau}_{r} y_{r o}>\left(1-\widehat{\Gamma}^{*}\right) \tau_{r} \widetilde{\tau}_{r} y_{r o}, & r \in \mathbf{O} \\
\sum_{j=1, j \neq o}^{n} \lambda_{j}^{*} y_{r j} \geq y_{r o}, & r \notin \mathbf{O}
\end{array}
$$

Then $\left(\widehat{\Gamma}^{*}, \lambda_{j}^{*},(j \neq o)\right)$ is a feasible solution to model $(2)$. Based on the proof of the lemma we can fined the feasible solution $\left(\widetilde{\Gamma}, \lambda_{j}^{*},(j \neq o)\right)$ to $\operatorname{model}(2)$ such that $\widetilde{\Gamma}<\widehat{\Gamma}^{*}$, violating the optimality of $\widehat{\Gamma}^{*}$.

Corollary: Suppose $D M U_{o}$ is a frontier point. If $\delta_{i} \widetilde{\delta}_{i}>1+\Gamma^{*}, i \in \mathbf{I}$ and $\tau_{r} \widetilde{\tau}_{r}<1-\Gamma^{*}, r \in \mathbf{O}$ then $D M U_{o}$ will not remain as a frontier point.

Proof: The proof is similar to that of theorem 3 and is omitted.

\section{Conclusion}

The current paper discusses simultaneous data perturbations in all DMUs, where the efficiency of the test DMU is deteriorating while the efficiencies of the other DMUs are improving. Necessary and sufficient conditions for preserving a DMU's efficiency classification presented in Zhu(2001) are developed and improved when various data changes are applied to all DMUs. The region, for preserving a DMU's classification, that is greater than the region presented in $\mathrm{Zhu}(2001)$ is also calculated. 


\section{References}

[1] P. Andersen, N.C. Petersen, A procedure for ranking efficient units in data envelopment analysis, Management science, 39 (1993), 1261-1264.

[2] A. Charnes, W.W. Cooper, E. Rhodes, Measuring the efficiency of decision making units, European Journal of Operational Research, 2(1978), 429-444.

[3] A. Charnes, W.W. Cooper, R.M. Thrall, A structure for classifying and characterizing efficiencies and inefficiencies in DEA, Journal of productivity analysis, 2, (1991) 197-237.

[4] A. Charnes, S. Haag, P. Jaska, J. Semple, Sensitivity of efficiency classifications in the additive model of data envelopment analysis, International Journal of Systems Science, bf 23 (1992), 789-798.

[5] A. Charnes, J. Rousseau, J. Semple, Sensitivity and stability of efficiency classifications in data envelopment analysis, Journal of productivity analysis, 7 (1996), 5-18.

[6] L.M. Seiford, J Zhu, Sensitivity analysis of DEA models for simultaneous changes in all the data, Journal of the Operational Research Society, 49 (1998), 1060-1071.

[7] L.M Seiford, J. Zhu, Infeasibility of super efficiency data envelopment analysis models, INFOR, 37(2) (1999), 174-187.

[8] J. Zhu, Super efficiency and DEA sensitivity analysis in DEA, European journal of operational research, 129 (2001), 443-455.

Received: July 4, 2006 